\title{
Geographie in der Welt von heute - Möglichkeiten und Grenzen eines integrativen Faches
}

\section{Eckart Ehlers, Bonn}

Die letzten Jahre haben einen bemerkenswerten Paradigmenwechsel innerhalb der Wissenschaften allgemein, damit aber auch in der wissenschaftlichen Geographie hervorgebracht. Der Trend hin zu einer neuen Komplexität ist unverkennbar. Klimawandel - Desertifikation - Meeresspiegelanstieg - Bevölkerungsexplosion - Verlust der Biodiversität - aber auch: veränderte Produktions- und Konsumptionsmuster in einer globalisierten bzw. sich globalisierenden Welt diese Themen sind tief im Bewußtsein einer verunsicherten Öffentlichkeit verankert; sie sind aber auch und zugleich Gegenstand einer Vielzahl wissenschaftlicher Disziplinen geworden. Erd- und Biowissenschaften, Physik und Chemie, Ökonomie und Völkerrecht, Soziologie wie Politologie - sie alle befassen sich mit diesen Fragen; sie alle scheinen aber als Einzeldisziplinen immer weniger in der Lage, befriedigende Lösungen der komplexen Probleme und Zusammenhänge geben zu können.

Eine solche Feststellung gilt umso mehr, als unilineare Beschreibung und Deutungsversuche $-z$.B. des sog. Global Change durch weltweite Klimaerwärmung - nicht mehr greifen. Zunehmend werden transund multidisziplinäre - um nicht das geknechtete Wort «interdisziplinäre» zu bemühen -, in jedem Fall aber fächerübergreifende Erklärungsmuster notwendig. Wenn ich im folgenden einige Anmerkungen zur Thematik

«Geographie - Möglichkeiten und Grenzen eines integrativen Faches»

mache, so möchte ich mich auf einige grundsätzliche Bemerkungen zur Methodik und Theorie, aber auch zur Problematik des integrativen Ansatzes beschränken. Zugleich aber sei einleitend und uneingeschränkt betont, daß das Potential der Geographie als integrativem Fach nicht so sehr in seinem eigendefinierten Selbstverständnis, sondern in seiner Kooperationsfähigkeit mit anderen Fächern sowie der Akzeptanz seiner fachlichen Kompetenz durch diese zu sehen ist.

Hartmut LESER hat mehrfach und in verschiedenen Kontexten Modelle integrativer Ansätze zur landschaftsökologischen Geosystematik vorgelegt. Aus technischen wie auch wissenschaftlichen Gründen soll es genügen, auf die entsprechenden Abbildungen bei Leser (z.B. 1997, Abb. 63 u.v.a.) oder bei anderen Autoren (z.B. Klug \& LANG 1983) und deren Erklärungsansätze $\mathrm{zu}$ verweisen. Wenn man unterstellt, daß solche oder ähnliche Modelle alle Voraussetzungen einer naturwissenschaftlich fundierten Modellbildung erfüllen, nämlich Vollständigkeit, Meßbarkeit, Quantifizierbarkeit sowie Überprüfbarkeit usw., dann steht der leicht zu beeindruckende Anthropogeograph staunend und fast neidisch vor dem Integrationspotential landschaftsökologischer und geosystemarer Regelkreise, Interdependenzen und systemimmanenter Wechselbeziehungen. Ich vermag nicht zu beurteilen, ob und inwieweit die z.B. bei Leser (1997, Abb. 63) oder an anderen Stellen angesprochenen Kompartimente systemischer Entitäten quantifizierbar und operationalisierbar und ihre Interaktionen empirisch nachvollziehbar sind. Tatsache indes scheint zu sein, daß Landschaftsökologie und Geosystemlehre ganz offensichtlich Zusammenhänge des physischen Landschaftshaushalts integrativ zu erfassen vermögen. Die entscheidende Frage indes bleibt, ob sie in einer Zeit, in der die «human domination of the Earth's ecosystems» unbestreitbar und an Bedeutung gewinnend ist, unsere aktuellen Umweltprobleme zu lösen in der Lage sind. Klimawandel - Desertifikation - Meeresspiegelanstieg - Landschaftsdegradationen - Verlust der Biodiversität: sie sind, wie wir heute wissen, in entscheidendem Maße vom Menschen beeinflußt. Die Frage also stellt sich: wie können wir den Menschen als Verursacher globaler Umweltveränderungen in Modelle und Vorhersagen bzw. Szenarios des Umweltwandels einbinden? Anders ausgedrückt: gibt es überhaupt humanökologische Modelle - und wenn $\mathrm{JA}$, wie lassen sich diese in natur- wie sozialwissenschaftliche Gesamtmodellierungen einfügen?

Wir alle wissen, daß die Humanökologie ein weites und diffuses Feld ist und nicht - wie ganz offensichtlich Bio- oder Geoökologie - über ähnlich vielfältige und differenzierte Modellansätze verfügt. Es wäre ganz zweifellos reizvoll, die Bandbreite humanökologischer Denkansätze sowie Möglichkeiten und Grenzen ihrer Modellierung auszuloten. Allein schon der Versuch, klare und eindeutige - ganz zu schweigen von allgemein akzeptierten! - Definitionen des Begriffes «Humanökologie» zu benennen, ist fast unmöglich.

Der vorliegende Beitrag war Thema des Symposiums «Anwendung und Perspektiven einer integrativen Wissenschaft» anlässlich des 60. Geburtstages von Prof. Dr. Dr. h.c. HaRTMUT LESER, veranstaltet am 2. Dezember 1999 in Basel. 
Weder die von Glaeser \& Teherani-KrönNer (1992) beziehungsweise von ScHMID (1994) herausgegebenen Sammelbände und die in ihnen enthaltenen Beiträge noch das von Nentwig (1995) verfaßte Lehrbuch, oder andere einschlägige Publikationen der letzten Jahre, z.B. Kilchenmann \& Schwarz (Hrsg.) (1992), Meinberg (1995) sowie der von Honari \& BOLEYN (1999) herausgegebene und insbesondere auf gesundheitsökologische Probleme ausgerichtete Sammelband vermitteln ein klares Gegengewicht gegen bio- oder geoökologische Modellansätze. Zu den immer noch konkretesten, wenngleich nicht operationalisierbaren Erklärungsansätzen dürfte GLAESERs Schema aus dem Jahre 1989 zählen, in dem zumindest der Versuch einer Beschreibung der Humanökologie gewagt wird (Abb. 1).

Die Feststellungen allerdings, daß die Humanökologie «als Grundlagendisziplin zwischen Natur- und Sozialwissenschaften steht» (GLAESER 1989: 28), sie «nicht als etablierte Fachwissenschaft im Rahmen der herkömmlichen, die Aufgaben zerschneidenden universitären Wissenschaftseinteilung zu sehen» (ebda.) und allenfalls «ein die Disziplinen vereinigendes Inter-

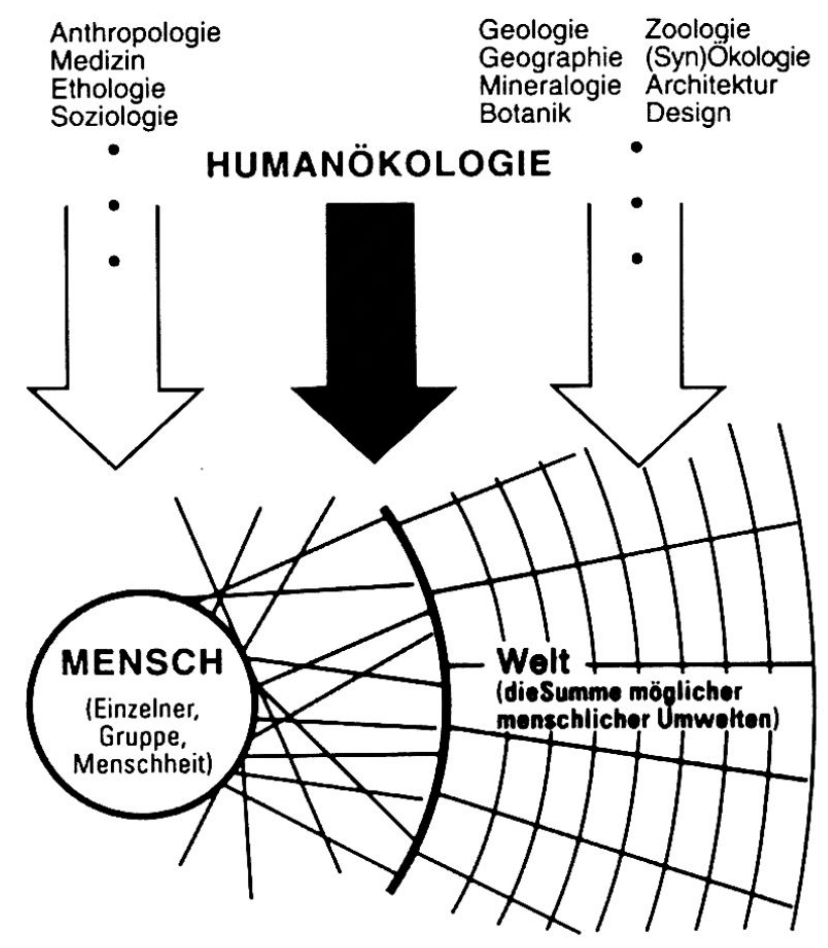

Abb. 1: Grundmuster humanökologischer MenschUmwelt-Beziehungen (nach GLAESER 1989, Abb. 2) Basic pattern of anthropo-ecological human-environment relationships (after GLAESER 1989, Fig. 2)

Schéma des relations homme-environnement du point de vue de l'écologie humaine (d'après GLAESER 1989, fig. 2) esse» sei (ebda.), zeigen die methodologische Problematik und die theoretische Unverbindlichkeit des humanökologischen Ansatzes, auch wenn der gleiche Autor die Humanökologie wenig später (Glaeser 1992:201) explizit als «Lehre von den Mensch-UmweltInteraktionen» bezeichnet. Insofern dürfte - wie noch zu zeigen sein wird - ENZENSBERGERS sehr distanzierter Definitionsversuch der Humanökologie gerade vor dem Hintergrund der Frage nach dem Integrationspotential des Faches Geographie von besonderer Bedeutung sein:

«Die Humanökologie ist zunächst einmal eine hybride Disziplin, in der natur- und sozialwissenschaftliche Kategorien und Methoden nebeneinander her angewandt werden müssen, ohne da $ß$ die Weiterungen, die sich hieraus ergeben, theoretisch in irgendeiner Weise geklärt wären» (ENZENSBERGER 1973: 1).

Des ungeachtet gibt es natürlich auch für die Rolle des Menschen im globalen Umweltgeschehen integrative Ansätze, die indes wesentlicher Elemente der naturwissenschaftlichen Modellbildung entbehren. So ist zum Beispiel das «Social Process Diagram» der Human Dimensions of Global Environmental Change (Abb. 2) nicht nur als offenes System konzipiert, sondern auch mit einer Reihe von Variablen ausgestattet, die sich den Kriterien der Vollständigkeit Meßbarkeit und Quantifizierbarkeit - z.T. auch der Logik und Überprüfbarkeit - teilweise, weitgehend oder gar ganz entziehen. Oder in den Worten eines der Autoren dieses Prozeßdiagramms:

"Research on this topic must consider human activities both as they contribute to and as they are affected by global environmental change. The human activities that interact with the Earth's natural systems are driven by three fundamental factors: the number of human beings and their distribution around the globe; human needs and desires, as conditioned by psychological, cultural, economic, and historical factors, which provide individuals and societies with motivations to act; and the cultural, social, economic, and political structures and institutions and norms and laws that shape and mediate their behaviour» (JACOBSON \& PRICE 1990: 13).

Das damit angesprochene Nebeneinander quantitativ faßbarer und qualitativ wirksamer Faktoren macht nicht nur Modellierungen innerhalb des in Abb. 2 angesprochenen Systems schwierig, sondern mehr noch Ansätze der Integration von natur- und sozialwissenschaftlichen Daten und Steuerungsgrößen. Vor diesem Hintergrund überrascht denn auch nicht, daß Sozialwissenschaftler ganz explizit von dem «social dilemma» sprechen, dem sie selbst unterworfen sind. Und selbst innerhalb vermeintlich homogener Wissenschaften wie z.B. der Ökonomie gehen die Auffassungen darüber, ob und inwieweit nur lokale oder globale ökonomische Modelle bestehenden oder zu erwartenden Realitäten gerecht werden, offensichtlich weit auseinander. 


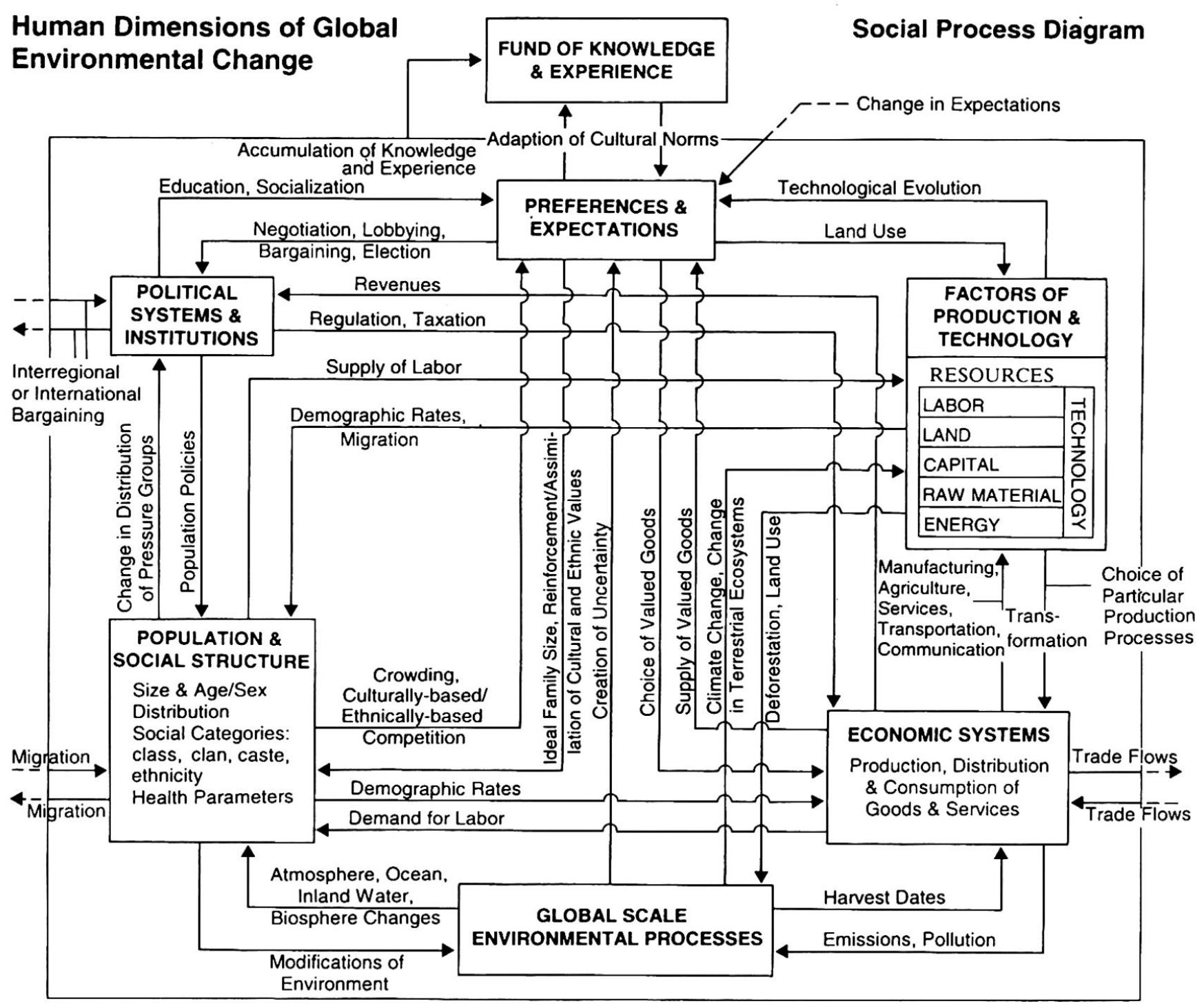

Note: This diagram shows representative levels for connections between building blocks

Abb. 2: Anthropogene Ursachen globaler Umwelttrends - Prozessdiagramm (Quelle (verändert nach): ConsoRtium for International Earth Science Information Network, Mai 1992); Graphik: L. BaumanN

Human dimensions of global environmental change - social process diagram (Source (changed according to): Consortium for International Earth SCIENCE Information Network, May 1992); illustration: L. BaumanN Les dimensions humaines du changement environnemental global - schéma du processus social (Source (modifié d'après): Consortium for International Earth Science Information Network, mai 1992); graphique: L. BauMANN

Mit dem «social dilemma» der Sozialwissenschaften ist auch das Dilemma der Geographie als einem integrativem Fach offenkundig: die Geographie als Brückenfach zwischen den Natur-, Geistes- und Sozialwissenschaften scheint abermals gefangen in seinem eigenen Selbstverständnis. Das zunehmend gefragte Integrationsbedürfnis wissenschaftlicher Erkenntnisse geht mitten durch das Fach hindurch und läßt die alte, ja schon traditionelle Dichotomie der Geographie als Natur- und Sozialwissenschaft erneut und anscheinend unvereinbar aufbrechen. Diese Kluft ist um so schmerzlicher als gerade die der Geographie so nahestehenden Forschungsfelder Umweltforschung, Erdsystemforschung und/oder Umweltmanagement integratives Denken und wissenschaftliche Integrationsfähigkeit verlangen.

Wie also ist das Dilemma der Geographie als einem integrativen Fach auf dem Gebiet der globalen Umweltforschung zu beheben und die postulierte Stärke des Faches - nämlich seine Integrationsfähigkeit - zu nutzen und zu fördern? Analog zu der in den letzten 
Jahren zu beobachtenden Abkehr von Globalmodellen des Umweltwandels (d.h. vor allem des Klimawandels!) und der Hinwendung zu praxis- und anwendungsorientierten Problemlösungen auf lokalen und/oder regionalen Maßstabsebenen (Umweltmanagement!) zeichnet sich $\mathrm{ab}, \mathrm{da} \beta$ holistisch-integrative Umweltforschung in Zukunft von folgenden Prämissen geleitet sein wird:

- eine begrenzte und klar definierte Problemstellung, die sich

- nicht an disziplinären Eigeninteressen, sondern an politisch/gesellschaftlich objektiven Fragestellungen orientiert, die sich

- in einem begrenzten räumlichen Bezugsrahmen abspielt, der zudem

- die gleichzeitige und aufeinander abgestimmte Kooperation verschiedenster Disziplinen erfordert mit dem

- Ziel einer Lösung des zuvor definierten Problems in einer gegebenen Zeit.

Die entscheidende Frage für echte integrative Forschung scheint zu lauten: was können Disziplinen mit ihren spezifischen Forschungsperspektiven und -methoden zur Lösung eines klar definierten Problems beitragen? Oder wie es ein Sozialwissenschaftler formulierte: «It is a political concern that integrates» (vgl. EHLERS 1999: 96). Mit anderen Worten: nicht disziplinäres Selbstverständnis um jeden Preis, fachspezifische Abkapselung und Nabelschau, sondern fächerübergreifende Kooperation mit dem Ziel einer Problemlösung im transdisziplinären Dialog und Austausch sind erfolgversprechend und zielführend. Eine solche generelle Feststellung gilt in ganz besonderer Weise für die komplexen Probleme globaler Umweltveränderungen. Daß dabei natur- und sozialwissenschaftliche Disziplinen in besonderer Weise auf Kooperation und Ko-Disziplinarität angewiesen sind, macht globale Umweltforschung über die konkreten Problembewältigungen hinaus gerade für und innerhalb der Geographie zu einem Test für Möglichkeiten und Grenzen natur- und sozialwissenschaftlicher Zusammenarbeit und problemlösungsorientierter Integrationsfähigkeit. $\mathrm{Da} \beta$ ein solches Postulat um keinen Preis disziplinäre Forschung und Weiterentwicklung behindern oder beeinträchtigen soll, ist so selbstverständlich, daß ich es hier nur der Vollständigkeit halber erwähne. Im Gegenteil: kompetente Disziplinarität ist unabdingbare Voraussetzung für fächerübergreifende Akzeptanz.

Wo liegen nun die Möglichkeiten und Grenzen der Geographie als einem integrativen Fach? Ich möchte - ohne Anspruch auf Vollständigkeit! - eine einfache, sehr einfache Antwort geben, zugleich aber damit verbundene Dilemmata wie auch Überwindungsstrategien aufzeigen. Die zunächst etwas vordergründige Antwort lautet:

Möglichkeiten und Grenzen des Faches Geographie als einem Integrator liegen in seiner multidisziplinären Kooperationsfähigkeit bei umweltrelevanten Problemlösungen auf lokalen bis regionalen Maßstabsebenen.

Im Grunde geht es zunächst einmal um nichts anderes als darum, Leistungsfähigkeit und Akzeptanz der Disziplin im trans-, multi- oder gar interdisziplinären Kontext bei der Bewältigung umweltrelevanter Forschungsthemen und Problemlösungen zu beweisen. Tatsache ist, daß neben Umweltforschung als Zweck und Ziel wissenschaftlichen Selbstverständnisses Umweltmanagement zunehmend an Bedeutung gewinnt. Mensch und Gesellschaft, aber auch die internationale Staatengemeinschaft haben mit Rio 1992 und den UN-Konventionen zum Schutz der Ozonschicht, $\mathrm{zu}$ Klimaveränderungen, zu $\mathrm{CO}_{2}$-Ausstoß (Kyoto), zu Wüstenbildung und zu Biologischer Vielfalt die Ära bewußten Managements des Gesamtsytems Erde begonnen, zu einer Art des «ecological engineering».

Zwei Beispiele mögen verdeutlichen, worum es bei dem Postulat «multidisziplinärer Kooperationsfähigkeit bei umweltrelevanten Problemlösungen auf lokalen bis regionalen Maßstabsebenen» geht:

Beispiel 1 ist das vom Deutschen Nationalen Komitee vorgeschlagene Projekt AQUA (Availability-QualityAllocation of Water). Das inzwischen vom Bundesministerium für Bildung und Forschung (BMBF) unter dem Titel GLOWA (Globaler Wasserkreislauf) öffentlich ausgeschriebene und für integrative Antragstellungen publizierte Dokument versteht sich dabei explizit als ein Beitrag zur Förderung disziplinärer Forschung. Es postuliert u.a. das Ziel, am Beispiel des globalen Wasserkreislaufs die «wissenschaftlichen Grundlagen für ein nachhaltiges, zukunftsfähiges Management von Ökosystemen und Gesellschaften» bereitzustellen. Dabei sollen aber zugleich «integrierte Forschungsansätze, die das System Erde als Ganzes und in allen Facetten berücksichtigen" gefördert werden sowie die Zusammenführung sektoraler Forschung und Entwicklung und schließlich auch die Vernetzung von Institutionen und Disziplinen.

Das vom Nationalkomitee und von den in ihm vertretenen natur- wie sozialwissenschaftlichen Disziplinen erarbeitete Rahmenkonzept ist eine Integrationsmatrix (Abb. 3), die durch die Kombination unterschiedlicher ökologischer Faktoren und Kernthemen und ihre vergleichende Untersuchung in funktional wie ökologisch unterschiedlichen Wassereinzugsgebieten in doppelter Hinsicht Möglichkeiten zu holistischintegrativen Forschungsansätzen eröffnet: zum einen 


\begin{tabular}{|l|l|l|l|l|} 
EinfluBfaktoren \\
Kernthemen
\end{tabular}

Abb. 3: Integrationsmatrix der inter-/trans-/multidisziplinären Zusammenarbeit von Natur- und Sozialwissenschaften an konkreten Fallbeispielen (verändert nach unveröffentlichten Unterlagen des NATIONALEN KomiteES FÜR GLOBALE UMWELTFORSCHUNG 1998)

Integrational matrix of interdisciplinary, transdisciplinary and multidisciplinary cooperation between the natural and social sciences, based on specific case studies (changed according to unpublished papers of the NaTIONALES KomiteE Für GLOBALE UmWELTFORSCHUNG 1998)

Matrice d'intégration de la collaboration inter-, trans- et pluridisciplinaire entre les sciences naturelles et sociales, illustrée à l'aide d'exemples concrets (modifié d'après des documents non publiés du NaTIONALES KomiteE FÜR GLOBALE UMWELTFORSCHUNG 1998)

innerhalb einzelner Flußsysteme, zum anderen im Rahmen einzelprojektübergreifender und systemvergleichender Modellierung.

Beispiel 2 ist der internationalen und disziplinenübergreifenden Zusammenarbeit des IGBP (International Geosphere-Biosphere Program) und des IHDP (International Human Dimensions of Global Change Program) entnommen und betrifft das Forschungsprogramm LUCC (Land Use and Land Cover Change). Das zwischenzeitlich hinreichend bekannte und in ganz entscheidender (um nicht zu sagen: ausschließlicher)
Weise von Geographen entwickelte und getragene Programm umfaßt sowohl in regionalen als auch in inhaltlich-methodischen Kontexten jene großskaligen wie lokalen/regionalen Maßstabsebenen, die geographischen Kooperationsformen in fächerübergreifenden Kontexten und Zusammenhängen angemessen scheinen (Abb. 4). Die Kombination von «social driving forces» mit den «biophysical driving forces» als Ursachen des Umweltwandels auf verschiedenen räumlichen Maßstabsebenen (unit of production - landscape region) ist im Verbund mit Zeitskalen unterschiedlicher Länge ein schönes Beispiel komplexer und diszi- 
plinen-integrierender Umweltforschung (vgl. dazu auch IGBP-IHDP 1995, 1999). Sie beantworten also gleichsam die Frage nach den Integrationsmöglichkeiten und -fähigkeiten des Faches Geographie. Dabei sei betont, daß weder GLOWA noch LUCC unter das plakative Etikett des «Umweltmanagement « fallen; «katastrophenpräventive Ursachenforschung des Umweltwandels auf regionalen Maßstabsebenen» wird den Intentionen beider Projekte wohl eher gerecht.

Die beiden angedeuteten Möglichkeiten der Geographie als einem integrativen Fach sind zugleich gute Beispiele, um auf Grenzen bzw. noch ungelöste methodische und theoretische Probleme im Natur-, Geistesund Sozialwissenschaften verbindenden Dialog zu verweisen. Aus Zeitgründen seien diese Grenzen oder Dilemmata hier nur angedeutet, ohne an dieser Stelle vertieft zu werden.

Dilemma 1: Unterschiedliche Gegenständlichkeit. Es ist ein Gemeinplatz darauf zu verweisen, daß sich die Naturwissenschaften als experimentelle und weitgehend mathematisch fundierte Disziplinen verstehen, die sich meist instrumentell operierend mit der materiellen Realität der Erde und des Kosmos befassen und deren Gesetzlichkeiten zu erklären suchen. Die Sozialwissenschaften demgegenüber sind jene,

«die die menschliche Gesellschaft, gesellschaftliche Gruppen, einzelne Individuen in ihrer Beziehung zu anderen oder Einrichtungen und Institutionen von Gesellschaften sowie materielle und kulturelle Güter als Ausdruck des Zusammenlebens von Menschen zum Gegenstand haben» (BAYER \& STÖLTING 1989: 302).

Irritiert allein schon das von den Verfassern dieser Definition selbst eingeräumte Fehlen eines eindeutigen Gegenstandsbereiches, so kommen bislang unvereinbar erscheinende Forschungsziele innerhalb der Sozialwissenschaften sowie die Persistenz von «Schulen « und Ideologien als nur schwer zu überbrückende weitere Hindernisse hinzu. Schließlich tragen auch die große disziplinäre Varietät sowie disziplinäre Traditionen und Selbstverständnisse zur «Unübersichtlichkeit» bei. Unterschiedliche Gegenständlichkeit gilt somit einmal für den grundsätzlichen Gegensatz zwischen Naturwissenschaften einerseits und zwischen Geistesund Sozialwissenschaften andererseits. Er gilt genauso aber auch für differierende Gegenständlichkeiten innerhalb der großen Wissenschaftsfelder selbst. Diese «Zweiteilung einer naturwissenschaftlichen Eindeutigkeit und einer geisteswissenschaftlichen Vieldeutigkeit» (GRÄFRATH 1990, zitiert nach GROH \& GROH 1991: 153 ) ist ein Grunddilemma aller holistisch-integrativen Wissenschaftlichkeit, vor allem dann, wenn es gilt, die komplexen Wirkungs- und Ursacheverhältnisse von Sachwelten und Sinnwelten als untrennbar zusammengehörig zu betrachten. Und daß der Wandel unserer Umwelt in diesem komplexen Ursache- und
Wirkungsverhältnis gesehen werden muß, dürfte unbestritten sein.

Dennoch wäre es m.E. aber fatal, diese Unterschiedlichkeit zum Anlaß der Verweigerung der fächerübergreifenden Zusammenarbeit zu nehmen. Gerade die Beispiele GLOWA und LUCC haben gezeigt bzw. beweisen, daß - ausgehend von klaren Frage- und Problemstellungen und räumlich überschaubaren Dimensionen - problemlösende Zusammenarbeit bis hin zu prognostischen Modellierungen auf lokalen und regionalen Maßstabsebenen möglich und sinnvoll ist. Es gibt inzwischen viele zumindest theoretische Ansätze, die diese Brückenschläge wagen (zur theoretischen Diskussion vgl. dazu z.B. HrRSCH 1995, Meyer-Abich 1988). Praktisch ist es wohl so, daß Natur-, Geistes- und Sozialwissenschaften vereinende Kooperationsformen bislang ihre Wirksamkeit allenfalls bei Problemlösungen auf lokalen oder regionalen Maßstabsebenen haben beweisen können.

Dilemma 2: Kompatible Methodik. Die Frage kompatibler Methodik hängt naturgemäß aufs Engste mit der Gegenständlichkeit des Untersuchungsobjektes zusammen. Das Auseinanderdriften der Weltsichten und Weltbilder seit Renaissance und Aufklärung, das Mit-, Neben- und Gegeneinander anthropozentrischer wie physiozentrischer Erklärungsversuche und Sinngebungen sowie die vermeintliche Unvereinbarkeit natur-, geistes- und sozialwissenschaftlicher Fragestellungen und Theorien bedürfen der Überwindung. So hat der amerikanische Biologe E.O. WILSON (1998: 252) erst kürzlich postuliert:

«Die beiden großen Forschungsbereiche können nur profitieren, wenn ihre Methoden und Kausalerklärungen in Einklang gebracht werden».

$\mathrm{Ob}$ eine solche Forderung in absehbarer Zeit realistisch einlösbar ist, mag bezweifelt werden. Andererseits bedarf die globale Umweltforschung gerade angesichts der von Naturwissenschaftlern zunehmend angemahnten «human dimensions» einer solchen Kooperation zwischen Natur- und Sozialwissenschaftlern. Wenngleich es mir abermals kurzfristig praktikabler erscheint, fächerübergreifende Zusammenarbeit zunächst entlang der zuvor formulierten Frage: «Was können Disziplinen mit ihren spezifischen Forschungsperspektiven zur Lösung eines klar definierten Problems beitragen?» zu wagen, so erscheint unabdingbar, daß sich in absehbarer Zeit kompatible Forschungsmethodiken zumindest zwischen Teilen der Natur- und Sozialwissenschaften auf dem Gebiet der globalen Umweltforschung entwickeln müssen, wenn man wirklich Fortschritte auf dem Gebiet der Umweltforschung und des Umweltmanagements erzielen will.

Die großen globalen Umweltprogramme sind sich dieser Problematik durchaus bewußt. Das von WBGU 
entwickelte Grundschema globaler Umweltveränderungen (WBGU 1993) geht davon ebenso aus wie WCRP, IGBP oder IHDP (vgl. dazu EHLERS 1998, Fig. 3). Auf die Beispiele AQUA/GLOWA bzw. LUCC wurde bereits verwiesen. Auch in dem gerade vom IHDP entwickelten Programm «Institutional Dimensions of Global Environmental Change» geht es u.a. um das «Problem of Fit» (= Kompatibilität/ Kommensurabilität). Dort heißt es im Kontext der Ecosystem and Human System Linkages:

«The problem of fit revolves around one fundamental idea; it asserts that the effectiveness of social institutions is a function of the match between the characteristics of the institutions themselves and the characteristics of the biogeophysical systems with which they interact. The better the match or fit between an institution and the relevant biophysical system, the more effective the institution will be!» (IDGEC 1999: 45).

Dilemma 3: Problematik der Maßstabsebenen. «Globaler Wandel», «Globale Umweltforschung» oder «global change research»: die fast schon zu einem geflügelten Wort gewordene «Globalität» der Umweltforschung macht deren Ursprung in den anfangs weltumspannenden Fragestellungen der Klimamodellierung deutlich. Inzwischen hat sich indes zunehmend die Kenntnis durchgesetzt, daß potentiell weltweit gültige Modelle auf regionalen oder lokalen Maßstabsebenen versagen bzw. im Widerspruch zu deren Befunden stehen. Umgekehrt wissen wir heute, dass lokale oder regionale Verursachungen ihre Konsequenzen in glo-

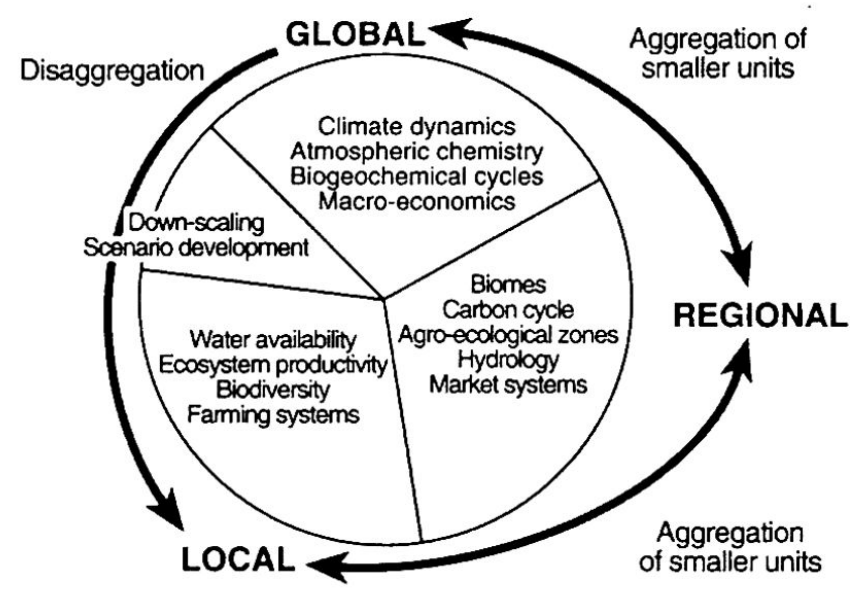

Abb.4: Integriertes Modellierungskonzept:Zur Verbindung unterschiedlicher regionaler Maßstabsebenen Integrated Modelling Framework: suitable for connecting different levels of scale (regional)

Cadre pour la modélisation intégrée: relation de divers niveaux d'échelles régionaux

(Quelle: LeEmans 1997: 65) balen Kontexten haben können. Aktuelles Beispiel dieser Interdependenzen sind die seit Jahren schwelenden Kohleflöze in China, die ungeahnte Auswirkungen auf den globalen $\mathrm{CO}_{2}$-Aussto $\beta$ und damit auf das Weltklima haben.

«Die brennenden Kohleflöze spiegeln eine gesundheitliche wie wirtschaftliche Katastrophe für China, aber vor allem schwelt hier eines der größten ökologischen Desaster der Erde. Wenn Kohle verbrennt, entsteht unweigerlich auch Kohlendioxid - jenes klimaschädliche Treibhausgas, das seit Jahren weltweit Wissenschaftler, Politiker und manchmal auch Industrielle zu drosseln versuchen. Meist vergeblich.

Auf Konferenzen zum Klimaschutz wird über Treibhausgas-Reduktionen bis zur zweiten Stelle hinter dem Komma gefeilscht. Absurd! Denn die an der Weltaufmerksamkeit vorbei flackernden Feuer unter China blasen jährlich so viel Kohlendioxid in die Luft wie die 42 Millionen Autos auf Deutschlands Straßen in vier Jahren. Das entspricht in etwa drei Prozent des globalen Treibhauseffekts. Geradezu katastrophal: China besitzt die größten Kohlereserven der Welt.

Die Brandmeldung lehrt zweierlei:

Weltweites Krisenmanagement existiert in dieser existenziellen Frage kaum. Und: Chinas brennende Kohlenflöze sind Wasser auf die Mühlen jener Experten, die vorschlagen, die begrenzten finanziellen Mittel erst einmal in umweltfreundliche Technik in der Dritten Welt zu investieren als in kostspielige Hightech-Lösungen vor der Haustüre. Das bringt global den maximalen Effekt. Schon morgen müssten die ersten Feuer gelöscht werden.

Egal, was es kostet» (WIEDLICH 1999).

Mit anderen Worten: die sich rasant ausweitende und inhaltlich wie thematisch vertiefende Umweltforschung reflektiert die Entwicklung dieser Forschung von einem zunächst globalen Problem- und Erklärungszusammenhang zu einer heute doch sehr viel differenzierteren Betrachtungsweise. Vor allem Sozialwissenschaftler unterschiedlichster Provenienz, zunehmend aber auch Naturwissenschaftler haben erkannt, daß Ursache und Wirkungen globalen Umweltwandels sehr häufig ihre Wurzeln und Konsequenzen auf lokalen oder regionalen Maßstabsebenen haben, während umgekehrt global wirksame Phänomene beträchtliche regionale oder lokale Auswirkungen haben können. Damit stellt sich zunehmend die Frage der Maßstäblichkeit in der globalen Umweltforschung sowie der Kompatibilität lokal, regional und/ oder global ermittelter Daten und ihrer Interpretationen. Entsprechende Methoden sind erst ansatzweise entwickelt. Andererseits verspricht die Vernetzung lokaler, regionaler und globaler Forschungsansätze nicht nur neue und erheblich ausgeweitete Erkenntnisse, sondern dürfte zugleich, insbesondere bei großmaßstäblicher Umweltforschung, die Chancen disziplin-übergreifender Kooperationen erhöhen (Abb.4). 
Der notgedrungen grobe Überblick zum allgemeinen Thema «Anwendung und Perspektiven einer integrativen Wissenschaft» zeigt, daß die globale Umweltforschung und die komplexen Mensch-Umwelt-Beziehungen gerade für das Fach Geographie in seiner potentiell integrierenden Mittlerstellung zwischen Natur-, Geistes- und Sozialwissenschaften besondere Chancen haben. Voraussetzung für eine optimale Nutzung dieser Chancen indes scheint mir zu sein, daß neben dem «Generalisierungspotential» und dem «Generalistenstatus» der Geographie ein engagiertes und von den Nachbardisziplinen anerkanntes fachwissenschaftliches Spezialistentum steht, das spezielle Problemlösungskompetenzen mit fachübergreifender Integrationsfähigkeit vereint. Im übrigen sei aber auch dieses mit allem Nachdruck betont: globale Umweltforschung ist naturgemäß nur ein Themenbereich, in dem das Fach Geographie die Grenzen und Möglichkeiten seiner Integrationsfähigkeit ausloten sollte. Der von Hartmut LESER u.a. vertretene landschaftsökologische Ansatz sowie das noch weitgehend unbeackerte Feld der Humanökologie wären weitere Beispiele für integrativ operierende Geographie (vgl. dazu schon z.B. BARRows 1923).

\section{Schlußbemerkungen}

Vor nicht allzu langer Zeit (1997) hat Wolf LEPENIES die Wiederbelebung einer «scholarship of engage-

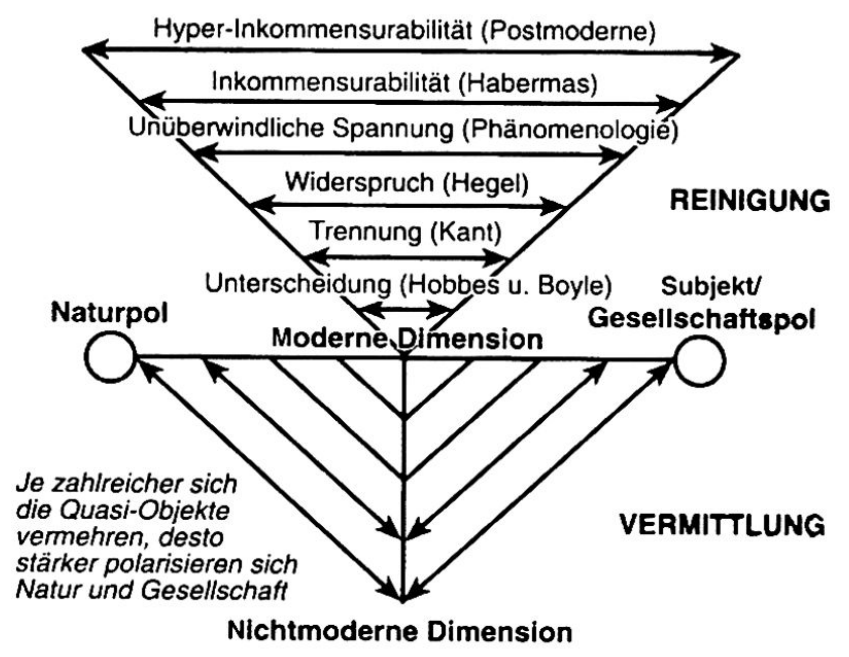

Abb. 5: Das moderne Paradox wissenschaftlicher Forschung zwischen Natur- und Sozialwissenschaft (nach Latour 1998, Abb. 6)

The modern paradox of scientific research in the natural and social sciences (after LATOUR 1998, Fig. 6)

Le paradoxe moderne de la recherche scientifique entre les sciences naturelles et les sciences sociales

(d'après LATOuR 1998, fig. 6) ment» eingefordert und dabei auch eine neue «Ideenpolitik», eine «Politik der Mentalitäten» reklamiert. Seine Feststellung, wonach

«Die strenge Beschwörung disziplinärer Identitäten mag noch einen Nutzen für die Verteilung knapper Ressourcen haben und altmodische akademische Hahnenkämpfe befeuern - geistespolitische Anregungen lassen sich auf diese Weise nicht mehr gewinnen» (LePENIES 1997: 93), gilt ganz sicherlich auch - und gerade dann! -, wenn es um die Frage nach der Integrationsfähigkeit von Wissenschaft(en) geht. Gerade das im Rahmen dieses Beitrags behandelte Beispiel der «Umweltforschung» ist ein Musterbeispiel für Möglichkeiten und Grenzen fächerübergreifender Diziplinarität.

Wissenschaftstheoretische und methodologische Diskurse während der letzten Jahre zeigten, daß die Frage nach der «Einheit des Wissens», d.h. nach holistischintegrativen Erklärungen und Deutungen unserer Welt und ihrer Teile wieder an Bedeutung gewinnt (vgl. E.O. WILson 1998). Vor allem seit Beginn der 90erJahre ist eine verstärkte Auseinandersetzung um das Neben- oder Miteinander von Sachwelt und Sinnwelt, von Objekt und Subjekt, von Natur und Mensch neu entbrannt (vgl. GroH \& Groh 1991, 1996; dort auch: weiterführende Literatur). So verweisen GroH \& Gron (1996: 89) bei der Diskussion um den anthropozentrischen versus den physiozentrischen Naturbegriff auf die zweifache Doppelnatur des Menschen: «...muß die Doppelnatur des Menschen als Naturwesen einerseits sowie als Kultur- und Vernunftwesen andererseits anerkannt werden». Analog zu dieser Doppelköpfigkeit des Naturwesens Mensch hat der französische Wissenschaftshistoriker und -philosoph B. Latour (1991/1998) mit Nachdruck und mit überzeugenden Argumenten darauf verwiesen, daß seit Bacon und Descartes bzw. Boyle und Новвеs das Auseinanderdriften der Wissenschaften als Ausdruck dieses Spannungsverhältnisses gesehen werden muß (vgl. Abb. 5).

Auch Latour argumentiert, daß die immer größere Zahl wissenschaftlicher Quasi-Objekte (Hybriden;vgl. dazu ZIERHOFER 1999), angesiedelt irgendwo zwischen dem immer weiter auseinanderklaffenden «Naturpol» einerseits und dem «Gesellschaftspol» andererseits der Überwindung im Interesse der Einheit von Mensch und Natur bedarf. Daß gerade die Umweltforschung ein Vehikel der Reformation wissenschaftlichen Denkens sein kann, hat jüngst H. MARKL (1998) betont. In einem programmatischen Aufsatz mit dem Titel «Umweltforschung als angewandte Naturwissenschaft» schreibt er wie folgt:

«...Umweltforschung als angewandte Naturwissenschaft ist also unvermeidlich ein Motor der Veränderung von Umweltverhältnissen, gerade indem sie bestimmte Umweltverhältnisse zu erreichen und zu erhalten bestrebt ist. ...Es ist ...ganz selbstverständlich, daß zwar naturwissenschaftliche Kenntnisse und Fähigkeiten eine bedeutende Rolle in 
der Umweltforschung und bei der Verwirklichung umweltbezogenen Handelns spielen, daß aber erstens geisteswissenschaftliche - philosphische, theologische und andere umfassend kulturwissenschaftliche - Beiträge unentbehrlich sind, um die Wertentscheidungen zu begründen, nach denen sich unser umweltbezogenes Verhalten ausrichten soll, und zweitens der umfassende Beitrag aller sozialwissenschaftlichen Disziplinen ebenso unersetzbar ist, um menschliches Umweltverhalten tatsächlich so zur Wirkung kommen zu lassen, wie es die Erfüllung einmal gesetzter Ziele erfordert...Umweltforschung ist daher, soll sie tatsächlich anwendungsorientiert sein, immer eine fast alle Wissenschaftsbereiche interdisziplinär vereinigende Forschung» (MARKL 1998: 135f.; vgl. auch MARKL 1986).

Gerade das letzte Zitat führt zu dem zurück, was einleitend als Postulat auch für die Möglichkeiten und Grenzen der Geographie (wie auch anderer Wissenschaften) als integrativem Fach gesagt wurde: nicht so sehr die selbstdefinierten Eigeninteressen eines Faches, sondern sein objektiv nachvollziehbares Vermögen zu problemlösender Wissenschaftskompetenz im Kontext fächerübergreifender Zusammenarbeit bestimmen die Integrationsfähigkeit wissenschaftlicher Disziplinen. Das gilt auch für die Geographie, die ihre diesbezüglichen Potentiale sehr viel mehr und sehr viel besser als bisher zur Geltung bringen sollte.

\section{Literatur}

BARrows, H.H. (1923): Geography as Human Ecology. - In: Annals Ass. Am. Geographers 13:1-14.

BAYER, O. \& E. STÖLTING (1989): Sozialwissenschaften. - In: Seiffert, H. \& G. RadnitzKy (Hrsg.): Handlexikon zur Wissenschaftstheorie. - München: 302-313.

CONSORTIUM FOR INTERNATIONAL EARTH SCIENCE INFORMATION NETwORK (CIESIN) (Hrsg.) (1992): Pathways of Understanding. The Interactions of Humanity and Global Environmental Change. - University Center, Michigan, May 1992.

EHLERS, E. (1998): Geographie als Umweltwissenschaft. - In: Die Erde 129:333-349.

EHLERS, E. (1999): Geosphäre-Biosphäre-Anthroposphäre: Zum Dilemma holistischer globaler Umweltforschung. - In: DitrmanN, A. \& A.-J. Wunderlich (Hrsg.): Geomorphologie und Paläoökologie (W. Andres-Festschrift). $-=$ Frankfurter Geowissenschaftliche Arbeiten Serie D. Physische Geographie 25: 87-104.

ENZENSBERGER, H.M. (1973): Zur Kritik der politischen Ökologie. - In: Kursbuch, 33, Ökologie und Politik oder Die Zukunft der Industrialisierung. - Berlin:1-42.

Glaeser, B. (1989): Entwurf einer Humanökologie. In: Glaeser, B. (Hrsg.): Humanökologie. Grundlagen präventiver Umweltpolitik. - Opladen: Westdeutscher Verlag: 27-45.

GlaESER, B. (1992): Natur in der Krise? Ein kulturelles Mißverständnis. - In: GAIA 1: 195-203.
Glaeser, B. \& P. Teherani-Krönner (Hrsg.) (1992): Humanökologie und Kulturökologie. - Opladen:Westdeutscher Verlag.

GROH, R. \& D. GROH (1991): Weltbild und Naturaneignung. Zur Kulturgeschichte der Natur. - Fankfurt am Main: Suhrkamp.

GroH, R. \& D. Groh (1996): Die Außenwelt der Innenwelt. Zur Kulturgeschichte der Natur 2. - Frankfurt am Main: Suhrkamp.

Hirsch, G. (1995): Beziehungen zwischen Umweltforschung und disziplinärer Forschung. - In: GAIA 4: 302-314.

Honari, M. \& T. Boleyn (Hrsg.) (1999): Health Ecology. Health, Culture and Human-Environment Interaction. - London, New York: Routledge.

IDGEC (1999): Institutional Dimensions of Global Environmental Change. Science Plan (IHDP Report 9) - Bonn.

IGBP-IHDP (1995): LUCC - Land Use and Land Cover Change. Science/Research Plan. StockholmGeneva (IGBP Report 35 / HDP Report 7).

IGBP-IHDP (1999): LUCC - Land Use and Land Cover Change. Implementation Strategy. StockholmBonn (IGBP Report 48 / IHDP Report 10).

JACOBSON, H. \& M.F. Price (1990): A Framework for Research on the Human Dimensions of Global Environmental Change. - Paris: International Social Science Council with the Cooperation of UNESCO.

Kilchenmann, A. \& C. Schwarz (Hrsg.) (1991): Perspektiven der Humanökologie. - Berlin u.a.: SpringerVerlag.

KLUG, H. \& R. LANG (1983): Einführung in die Geosystemlehre. - Darmstadt: Wiss. Buchgesellschaft.

Latour, B. (1998): Wir sind nie modern gewesen. Versuch einer symmetrischen Anthropologie. - Frankfurt am Main: Fischer Taschenbuch-Verlag - Französische Originalausgabe (1991): Nous n'avons jamais été modernes. Essai d'anthropologie symétrique. - Paris: La Découverte.

Leemans, R. (1997): LUCC Report Series No 1: Open Science Meeting Proceedings. - LUCC International Project Office, Barcelona: 65.

LEPENIES, W. (1997): Benimm und Erkenntnis. - Frankfurt am Main: Suhrkamp.

LESER, H. (1997): Landschaftsökologie. (4. Aufl.) Stuttgart: UTB-Ulmer.

MARKL, H. (1986): Natur als Kulturaufgabe - Über die Beziehung des Menschen zur lebendigen Natur. Stuttgart: Deutsche Verlags-Anstalt.

MARKL, H. (1998): Wissenschaft gegen Zukunftsangst. - München, Wien: C. Hanser Verlag. Darin insbes. Kap.II/3: Umweltforschung als angewandte Naturwissenschaft: 127-146.

Meinberg, E. (1995): Homo oecologicus. Das neue Menschenbild im Zeichen der ökologischen Krise. Darmstadt: Wiss. Buchgesellschaft.

Meyer-Abich，K.M. (1988): Wissenschaft für die 
Zukunft - Holistisches Denken in ökologischer und gesellschaftlicher Verantwortung. - München: C.H. Beck.

Nentwig, W. (1995): Humanökologie. Fakten - Argumente - Ausblicke. - Berlin, Heidelberg, New York: Springer-Verlag.

ScHмID, J. (Hrsg.) (1994): Bevölkerung - Umwelt Entwicklung. Eine humanökologische Perspektive. Opladen: Westdeutscher Verlag.

Wiedlich, W. (1999): China macht Weltklima. - In: General-Anzeiger Bonn, 8. Dezember 1999; Kommentar zum Artikel «Im Boden schwelt eine Katastrophe»: 47.

WiLson, E.O. (1998): Consilience. The Unity of Knowledge. - New York: Alfred A. Knopf; Deutsche Ausgabe (1998): Die Einheit des Wissens. - Berlin: Siedler.

Zierhofer, W. (1999): Geographie der Hybriden. - In: Erdkunde 53: 1-13.

\section{Weiterführende Literatur}

BARGATZI, T. (1986): Einführung in die Kulturökologie - Umwelt, Kultur und Gesellschaft. - Berlin: Reimer.

Kessler, H. (Hrsg.) (1996): Ökologisches Weltethos im Dialog der Kulturen und Religionen. - Darmstadt: Wiss. Buchgesellschaft.

Lubchenco, J. (1998): Entering the Century of the Environment: A New Social Contract for Science. Science 279: 491-497.

Meyer-Abich, K.M. (1997): Praktische Naturphilosophie. Erinnerung an einen vergessenen Traum. München: C.H. Beck.

Schellnhuber, H.-J. \& V. Wenzel (Hrsg.) (1998): Earth System Analysis. Integrating Science for Sustainability. - Berlin u.a.: Springer-Verlag.

SiEfERLE, R.P. (1997): Rückblick auf die Natur. Eine Geschichte des Menschen und seiner Umwelt. München: C.H. Beck.

UNFCCC (United Nations Framework Convention on Climate Change) (1998): The Kyoto Protocol to the Convention on Climate Change. - Bonn (UNFCCC), Geneva (UNEP/ICU).

Vitousek, P., Mooney, H., Lubchenco, J. \& J. Melillo (1997): Human Domination of Earth's Ecosystems. In: Science 277: 485-499.

WBGU (Wissenschaftlicher Beirat der Bundesregierung Globale Umweltveränderungen) (1993): Welt im Wandel: Grundstruktur globaler Mensch-UmweltBeziehungen. - Bonn: Economia Verlag.
Zusammenfassung: Geographie in der Welt von heute Möglichkeiten und Grenzen eines integrativen Faches Die Ausführungen dieses Beitrags plädieren für eine Mittlerrolle der Geographie im Grenz- und Überschreitungsbereich von Natur-, Geistes- und Sozialwissenschaften. Gerade im Bereich des globalen Umweltwandels sowie einer damit zusammenhängenden Neubestimmung der Mensch-Umweltbeziehungen wird der Geographie eine Schlüsselfunktion im Hinblick auf eine neue «Einheit des Wissens» zuerkannt.

Résumé: La géographie dans le monde d'aujourd'hui - perspectives et limites d'une discipline intégrative

Cet article défend le rôle de médiateur de la géographie dans la zone frontière entre les sciences naturelles, humaines et sociales. C'est justement dans les domaines du changement environnemental global et de la redéfinition des relations entre l'homme et l'environnement qu'il implique que la géographie se voit attribuer une fonction clé en ce qui concerne une nouvelle «unité du savoir».

\section{Summary: Geography Today - Chances and limits of an integrative discipline}

This article calls on Geography to take up a mediating role in the interface between the natural, social and humanistic sciences. Global environmental change and the increased focus on human and environmental interrelations make Geography a key player in inter- and transdisciplinary research and the efforts to achieve «Unity of Sciences».

Prof. Dr. Eckart Ehlers, Institut für Wirtschaftsgeographie der Universität Bonn, Meckenheimer Allee 166, D-53115 Bonn.

e-mail:ehlers@joyce.giub.uni-bonn.de 